Aus der dermatologischen Abteilung des städt. Krankenhauses zu Dortmund (leitender Arzt: Oberarzt Dr. J. Fabry).

\title{
Nachtrag zu der Arbeit von 0. Müller: „Ein Fall von Pityriasis rubra Hebrae mit Lymphdrüsentuberkulose. ${ }^{\left({ }^{1}\right)}$
}

Von

\author{
Dr. Hermann Fabry, \\ I. Assistenzarzt der Abteilung.
}

(Mit einer Abbildung im Texte.)

O. M üller veröffentlichte in diesem Archiv, Bd. LXXXVII, pag. 255, einen Fall von Pityriasis rubra Hebrae mit Lymphtuberkulose der Achseldrüsen. Wir haben nun diesen Fall bis zum 7. Februar 1908 beobachtet, wo er ad exitum kam und sind daher in der Lage, sowohl das klinische Krankheitsbild zu vervollständigen, als auch durch den Sektionsbefund und anschlieBende histologische Untersuchungen den pathologischanatomischen Teil der Arbeit zu ergänzen.

Wenn wir an die Beobachtungszeit von O. Mäller anschließen, so konnten wir feststellen, daß das Allgemeinbefinden der Patientin Ende 1907 immer schlechter wurde. Subjektiv fühlte Patientin sich sehr elend, der Stuhlgang wurde träge und erfolgte auf Brustpulver oder Darmirrigationen alle 3-4 Tage einmal. Dagegen gingen die entzündlichen Hauterscheinungen zurück. Zwar kam es im November noch zweimal zu akuten erythematösen Gesichtsreizungen. Außerdem beteiligte sich besonders die Kopfhaut an diesen Reizerscheinungen, indem sie sich mit Schuppen bedeckte, deren übermäßige Neubildung nur durch Ölverbände in Schranken zu halten war. Die Erscheinungen im Gesichte und auf dem Kopfe, die unter leichtem Fieber verliefen, gingen jedesmal unter Vaseline-Verbänden in wenigen Tagen zurück. Die Haut des Körpers wurde im Laufe der

1) Archiv. Bd. LXXXVII. 
Zeit stetig gespannter und atrophischer, besonders über den Gelenken. Die hellen Flecke, die bereits, wie von $M$ üller erwähnt, immer deutlicher zutage traten, hoben sich scharf gegen ihre stärker pigmentierte Umgebung $a b$, besonders auf der Streckseite der Oberschenkel.

Seit Oktober entwickelte sich auf dem linken Auge eine diffuse Hornhauttrübung, die allmählich zur völligen Verdichtung der linken Hornhaut fübrte. Im Januar 1908 konnte Patientin mit dem linken Auge nicht mehr sehen. Ende Januar begann auch die rechte Hornhaut sich zu trüben.

Mitte Januar 1908 stellte sich Urinverhaltung ein. Der Urin mußte von jetzt ab täglich mit dem Katheter entleert werden.

Die Spannung der Haut über den Gelenken war inzwischen so stark geworden, daß Patientin sich nur noch mit Mühe im Bett bewegen konnte. Unter dem Kreuzbein begann sich ein Dekubitus trotz Luftkissen und vorsichtiger Lagerung zu entwickeln. Ein Dauerbad stand leider nicht zur Verfügung.

Am 7. Februar 1908 erfolgte der Exitus infolge Pneumonie.

Der Sektionsbefund, der uns von Herrn Dr. John, Sekundärarzt der inneren Abteilung, überlassen wurde, war folgender:

$\ddot{A}$ u Bere Besichtigung. Haut welk, leicht, bräunlich verfärbt, auf der Vorder- und Rückfäche des Rumpfes und der Extremitäten zahlreiche bis pfennigstückgroße weißliche Flecken. Keine fühlbaren Achseldrüsen-Ödeme beider Unterschenkel.

Bauchsitus. Flectura sigm. nimmt die ganze linke Bauchhälfte bis zum Nabel herauf ein, zwischen Leber und Bauchfell lagern mehrere Dünndarmschlingen. Peritoneum überall glatt und glänzend.

Brustsitus. Außer circumscripten, strangförmigen Verwachsungen des oberen seitlichen linken Unterlappens ohne path. Befund. Zwerchfellstand links IV. J. c. R., rechts III. J. c. R.

Lungen. Links. Oberfläche des Oberlappens sowie auch des Unterlappens bis auf die eine adbärente Stelle glatt und spiegelnd. Oberlappen von weicher Konsistenz, emphysematös gebläht, Unterlappen fühlt sich ziemlich derb an, auf dem Durchschnitt zwischen dunkelroten, auf der Schnittfäche vorragenden, derberen, nicht mehr lufthaltigen Partien hellere, graurot gefärbte, noch lufthaltige Bezirke. Auf Druck entleert sich aus der Schnittfläche aus einzelnen Lumina in den infiltrierten Bezirken ein gelbliches, eitriges Sekret. Der rechte Unterlappen bietet im wesentlichen denselben Befund wie der linke Unterlappen, nur fühlt er sich im ganzen nicht so derb an und auf der Schnittläche finden sich weniger zahlreiche, verdichtete, nicht mehr lufthaltige Bezirke. Im rechten Oberlappen finden sich mehrere nebeneinander liegende, hirsekorngroße, harte Knötchen, ebenso im linken Oberlappen ein vereinzeltes größeres, das auf dem Durchschnitt gleichmäßig grauschwarz aussieht. Bronchialdrüsen nur bis reichlich erbsengroß, von etwas derber Konsistenz, auf dem Durchsehnitt grauschwarz, mit grauweißen Zügen durchsetzt. 
Halsorgane und Thyreoidea ohne pathol. Befund. Oesophagus, Trachea ohne Befund. Keine Schwellungen der Halslymphdrüsen.

Herz leicht vergrößert infolge geringfügiger Dilatation; von schlaffer Konsistenz. Muskulatur braunrot mit Fett überlagert. Klappen obne Befund. Endokard enthält Einlagerungen kleiner, erbsengroßer, gelblicher, harter Plaques, sowohl in der Nähe der Mitral- und Trikuspidal-, wie auch über der Aortenklappe. Intima der Aorta gleichfalls stellenweise mit gelblichen Plaques bedeckt.

Milz etwas vergrößert. OberÄ̈che glatt, von derber Konsistenz, auf dem Durchschnitt gleichmäßig rot-fleischfarben mit deutlicher Trabekelzeichnung.

Neben ni eren beiderseits von normaler Größe ohne path. Befund.

Nieren beiderseits verkleinert. Kapsel verdickt, zwar etwas erschwert, aber ohne Substanzverlust abziehbar, Nierenoberfäche leicht böckrig, GröBe der einzelnen Höcker die eines Pflaumenkernes und darüber, zwischen den Höckern eingesunkene Partien. Konsistenz der Nieren etwa normal. Auf dem Durchschnitt Rinde deutlich verschmälert, Rindensubstanz, auch die zwischen den Markkegeln gelegene, mit gelblichen Fleckungen und Zügen durchsetzt.

Leber. Oberfäche glatt, Konsistenz normal, bietet auf dem Durchschnitt das Aussehen einer hyperämisch-fettigen (Muskatnuß-) Leber, mitten in der Substanz des rechten Lappens ein fast erbsengroßer, speckiggelblich-weißer Herd (zur mikrosknp. Untersuchung herausgeschnitten).

Gallenblase o. B. Gallengänge durchgängig.

Bauchspeicheldrüsen ohne Befund.

Darm. In der Darmschleimhaut, fast in ganzer Ausbreitung des Dünndarmes, Injektion der Gefäße, sonst ohne Befund.

Magen ohne Befund.

Mesenterium. Keine Drüsenschwellungen zu bemerken.

$B l$ a se. Blasenwand deutlich verdickt, Schleimbaut verdickt, besonders einzelne bläulich verfärbte, zum 'Teil mit einem fest haftenden gelblich-weißen Belag versehene Partien ragen deutlich hervor, dazwischen kleinere, hirsekerngroße und halblinsengroße, grau ausseheade, flache Erhebungen, am Blasenhals varikös erweiterte Venen. Adnexe zart, etwas atrophisch.

Uterus leicht vergrößert, im Fundus Uteri, der Hinterwand auf. liegend, eine haselnußgroße, schwammige, blaßrot aussehende Gew ebsbildung, sonst bietet der Uterus keinen path. Befund. Befund.

Gehirn. Geringfügiger Hydrocephalus. Sonst ohne jeglichen pathol.

Achseldrüsen. In der Haut der Achselhöhlengegend mehrere nebeneinander liegende straffe Narben. In der Achselhöhle selbst etwas vergrößerte Drüsen, die sich derb anfüblen und auf dem Durchschnitt grauschwarz aussehen, dazwischen sind grauweiße bis gelbliche, straff fibröse Gewebspartien zu bemerken, die in einem Fall über $1 / 3$ einer Drüse ausfüllen. 
Nach der Sektion wurden von uns verschiedene Organteile gehärtet und in Paraffin eingebettet.

Histologisch fand sich nun folgendes:

Die im Sektionsprotokoll erwähnten Knötchen der Oberlappen der Lunge waren derb fibröses Gewebe, in dem nur wenig Zellkerne lagen. Die Bronchialdrüsen waren ebenfalls von bindegewebigen Strängen durchzogen, während die eigentliche Drüsensubstanz geschrumpft war. Aber auch in ihnen fanden sich nirgends akut entzündliche Prozesse.

Der speckige Herd der Lebersubstanz erwies sich histologisch als ein Fibrom.

Die grauen flachen Erbebungen der Blasenschleimhaut, die schon mikroskopisch deutlich sichtbar waren, bestanden aus adenoidem Gewebe ohne entzündliche Erscheinungen und waren als Follikel anzusprechen.

Die Achseldrüsen waren sehr stark ron bindegewebigen, sklerosierenden Zügen durchsetzt, so daß das Drüsengewebe stark geschrumpft, zum Teil gänzlich atrophisch war. Aber auch hier nirgends zellige Infiltration, vor allem keine circumscripte Zellanhäufungen mit Riesenzellen.

Wenn wir auch nach obigem histologischen Befund nirgends im Körper einen aktiven tuberkulösen Prozeß gefunden haben, so müssen wir doch die vernarbten Herde in der Lunge und in den Bronchialdrüsen, vor allem aber die derbe Bindegewebswucherung der Achseldrüsen auf überstandene tuberkulöse Prozesse zurückzuführen, die in bindegewebige Vernarbung übergegangen sind, zumal von $\mathrm{M} \ddot{\text { üller}}$ in dem Eiter der rechten Achseldrüsen Tuberkelbazillen nachgewiesen wurden.

Müller erwähnt in seiner Arbeit bezüglich der Hauterscheinungen des Krankheitsbildes, daß sich auf der Haut pigmentlose Flecke gebildet hatten, die später an Deutlichkeit zunahmen, indem die umgebende Haut dieser weißen leukodermartigen Flecke sich stärker pigmentierte. Wir konnten beobachten, daß diese weißen Hautstellen bis zum Tode immer deutlicher wurden, besonders auf der Streckseite der Oberschenkel. Bei der Sektion wurden mehrere Hautstücke herausgeschnitten, die einen solchen Fleck mit umgebender stärker pigmentierten Haut umfaßten behufs Einbettung und histologischer Untersuchung. Wir fanden nun folgendes: 
Innerhalb der weißen Flecke war die Haut in toto verschmälert, die Hornschicht war sehr dünn, die Epidermispapillen fast verstrichen, an manchen Stellen nur angedeutet. Auch das Corium war geschrumpft und von diffuser Infiltration durchsetzt. Dieses Bild änderte sich am Rande der Flecke insofern, als die Epidermis dicker erscheint. Das Stratum malpighii läßt deutliche Zapfen erkennen, ist aber noch immer gegen eine normale Haut als verschmälert anzusehen. In der Basalschicht treten nun plötzlich reichliche Pigmentkörperchen auf. Die Pigmentkörperchen finden sich auch im Corium in zahlreichen Chromatophoren. Im Bau des Coriums sind sonst keine wesentlichen Veränderungen im Verbältnis zum Corium der hellen Flecke zu verzeichnen.

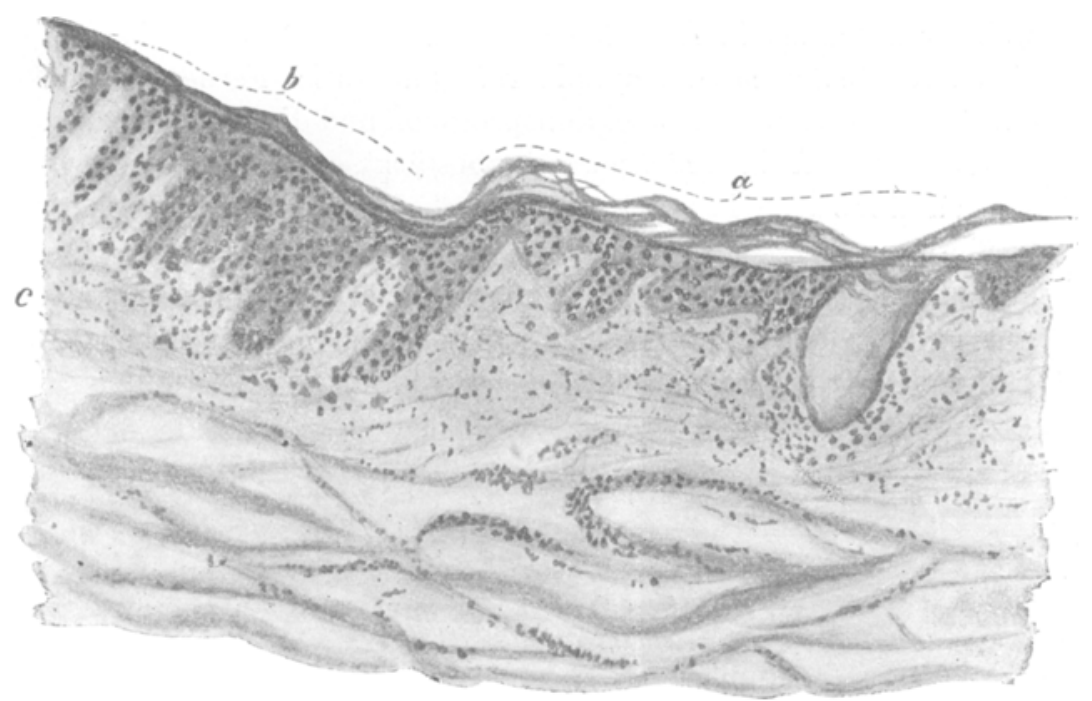

a) Rand eines pigmentfreien hellen Fleckes, Epidermispapillen verstrichen.

b) Pigmentierte Umgebung eines hellen Fleckes. Pigment der Basalschicht. Chromatophoren in der Cutis (c), Epidermispapillen deutlicher wie bei $a$ ).

Unsere Abbildung illustriert diese Verhältnisse sehr gut, als der Schnitt durch den Rand eines hellen Fleckes geführt, auf der einen Seite $(\boldsymbol{a})$ den pigmentfreien Fleck selbst, auf 
der andern Seite $(b)$ die pigmentierte Umgebung des Fleckes mit deutlichem Pigment der Basalschicht und des Coriums zeigt.

In den tieferen Schichten der Haut ist das Gewebe atrophiert. Man sieht sklerosierende Bindegewebszüge die Cutis und Subcutis durchsetzen. Talgdrüsen finden sich im Gegensatz zu den Schweißdrüsen nur wenig und zwar in atrophischem Zustand.

Wir haben also im allgemeinen eine allgemeine Atrophie der Haut vor uns. Außerdem herdweise Pigmentschwund, der sich makroskopisch in hellen Flecken repräsentiert.

Die weitere Beobachtung des Krankheitsbildes hat also gelehrt, daß unser Fall pathologisch-anatomisch aufzufassen ist als ein in der Cutis und Subcutis lokalisierter kleinzelliger Infiltrationsprozeß mit sekundärer Proliferation der Epidermis, welcher sekundär in Atrophie ausgeht. Diese Atrophie bezieht sich auf alle Schichten der Epidermis und Cutis. Ferner werden wir besonders durch das Sektionsprotokoll und die histologischen Untersuchungen in der Annahme bestärkt, daß, wie Jadassohn und D o u trele pont hervorgehoben haben, ein Zusammentreffen von Pityriasis rubra Hebrae mit Tuberkulose mehr Beobachtung geschenkt werden muß. In neuerer Zeit hat ja auch $\mathrm{Halle}$ aus der Lesserschen Klinik einen hierher gehörigen Fall im Archiv veröffentlicht. 